\title{
Overexpression of IQGAP1 promotes the angiogenesis of esophageal squamous cell carcinoma through the AKT and ERK-mediated VEGF-VEGFR2 signaling pathway
}

\author{
CHAO-HUI LI $^{1 *}$, XUE-JIAO SUN ${ }^{1 *}$, SHI-SHI NIU ${ }^{1}$, CHENG-YUAN YANG $^{1}$, YUE-PENG HAO ${ }^{1}$, \\ JUN-TING KOU $^{1}$, XIAO-ZHONG LI $^{2}$ and XIAO-XIA WANG ${ }^{1}$ \\ ${ }^{1}$ Department of Biochemistry and Molecular Biology, Shanxi Medical University, Taiyuan, Shanxi 030001; \\ ${ }^{2}$ Department of Emergency, Shanxi Provincial People's Hospital, Taiyuan, Shanxi 030012, P.R. China
}

Received March 16, 2018; Accepted July 3, 2018

DOI: 10.3892/or.2018.6558

\begin{abstract}
Angiogenesis is crucial for the progression of esophageal squamous cell carcinoma (ESCC). Anti-angiogenesis by targeting important molecules has been considered as one of the most promising and efficient strategy for cancer therapy. Recent studies have demonstrated that the IQ-domain GTPase activating protein 1 (IQGAP1) plays critical roles in tumorigenesis and cancer progression. We previously reported that IQGAP1 is overexpressed in ESCC, and IQGAP1 knockdown can decrease cell proliferation and metastasis ability in vitro and in vivo. However, the effects of IQGAP1 on the angiogenesis of ESCC and its underlying mechanisms remain unknown. In the present study, we found that IQGAP1 overexpression promoted tumor angiogenesis confirmed by human umbilical vascular endothelial cell (HUVEC) tube formation in vitro and chicken embryo chorioallantoic membrane (CAM) assay in vivo. Moreover, IQGAP1 overexpression in ESCC cells increased expression of vascular endothelial growth factor (VEGF) and phosphorylation of vascular endothelial growth factor receptor 2 (VEGFR2). Meanwhile, we found that levels of AKT and ERK phosphorylation were upregulated in IQGAP1-overexpressing cells. Importantly, IQGAP1-knockdown cells showed the opposing results. Furthermore, AKT and ERK inhibitors not only significantly
\end{abstract}

Correspondence to: Dr Xiao-Xia Wang, Department of Biochemistry and Molecular Biology, Shanxi Medical University, 56 Xinjian South Road, Taiyuan, Shanxi 030001, P.R. China

E-mail: wxiaoxia99007@126.com

Mr. Xiao-Zhong Li, Department of Emergency, Shanxi Provincial People's Hospital, 29 Shuangta Street, Taiyuan, Shanxi 030012, P.R. China

E-mail: lixiaozhong@126.com

${ }^{*}$ Contributed equally

Key words: IQGAP1, angiogenesis, esophageal squamous cell carcinoma, VEGF, VEGFR2 decreased VEGF expression and VEGFR2 phosphorylation in IQGAP1-overexpressing cells, but also abolished the pro-angiogenic effect of IQGAP1 overexpression on angiogenesis in the HUVEC tube formation and chicken embryo CAM assay. Taken together, this evidence confirms that IQGAP1 overexpression promotes tumor angiogenesis via the AKT and ERK-mediated VEGF-VEGFR2 signaling pathway in ESCC, and IQGAP1 may be an attractive therapeutic target for cancer anti-angiogenesis treatment.

\section{Introduction}

Esophageal cancer is one of the most aggressive and fatal gastrointestinal tract malignancies worldwide. Esophageal squamous cell carcinoma (ESCC) is the predominant histological type of esophageal cancer in China (1). Although clinical diagnostic and multidisciplinary therapeutic progress has been made, the overall prognosis of ESCC patients is still unfavorable due to rapid progression and metastasis. To date, the underlying mechanisms involved in the initiation and progression of ESCC are not fully understood. Therefore, a thorough understanding of the molecular mechanisms underlying the carcinogenesis and progression of ESCC is vital for discovering novel targets and innovative treatment strategies.

Angiogenesis, the process leading to the formation of new blood vessels from preexisting ones, is one of the major hallmarks of cancer, and is involved in the progression and growth of cancer $(2,3)$. Understanding the molecular mechanisms responsible for tumor angiogenesis can benefit cancer diagnosis and treatment. Thus, suppression of tumor angiogenesis offers a promising strategy for targeted therapy of cancer.

IQ-domain GTPase activating protein 1 (IQGAP1) is a member of a family of scaffolding proteins, which regulate distinct cellular processes including cell adhesion, proliferation, migration and other cellular functions through interacting with diverse proteins (4-6). Thus, IQGAP1 is as a critical integrator of cellular signaling pathways. Several studies have shown that IQGAP1 expression is increased in various cancer tissues, including colorectal carcinoma $(7,8)$, breast (9), ovarian (10), lung (11), pancreatic (12) and thyroid cancer (13). Furthermore, high expression of IQGAP1 promotes invasion 
and metastasis, and exhibits a significant correlation with poor patient prognosis (7-13). We reported that IQGAP1 is highly overexpressed in ESCC and the knockdown of IQGAP1 by small interfering RNA (siRNA) can decrease cell proliferation and metastasis ability in vitro and in vivo (14), indicating that IQGAP1 is a potential target for cancer treatment. However, the role of IQGAP1 in the angiogenesis of ESCC is not yet known.

In the present study, we investigated the role of IQGAP1 in regulating the angiogenesis of ESCC and explored its underlying molecular mechanisms. We report that IQGAP1 overexpression promotes angiogenesis of ESCC by the AKT and ERK-mediated vascular endothelial growth factor (VEGF)-vascular endothelial growth factor receptor 2 (VEGFR2) signaling pathway. These findings suggest an essential role of IQGAP1 in the angiogenesis of ESCC and provide novel insight into IQGAP1 as an attractive therapeutic target for cancer anti-angiogenesis treatment.

\section{Materials and methods}

Cell culture and stable transfections. Human ESCC cell lines EC9706 and KYSE150 were purchased from the Tumor Cell Bank of the Chinese Academy of Medical Sciences (Beijing, China). Human umbilical vascular endothelial cells (HUVECs) were purchased from the CHI Scientific Inc. (Jiangsu, China). The cells were maintained in Gibco Dulbecco's modified Eagle's medium (DMEM; Thermo Fisher Scientific, Inc., Waltham, MA, USA) supplemented with $10 \%$ fetal bovine serum (FBS; HyClone Laboratories; GE Healthcare Life Sciences, Logan, UT, USA), penicillin and streptomycin at 5\% $\mathrm{CO}_{2}$ and $37^{\circ} \mathrm{C}$ in a humidified incubator. The GFP-IQGAP1 overexpression and control plasmids were purchased from GeneCopoeia, Inc. (Rockville, MD, USA) and transfected into EC9706 cells. Non-specific control and IQGAP1 shRNA (short hairpin RNA) plasmids were purchased from Shanghai Genechem Co., Ltd., (Shanghai, China) and transfected into KYSE150 cells. Transfection of the plasmids was carried out using Invitrogen Lipofectamine 2000 transfection reagent (Thermo Fisher Scientific, Inc.) following the manufacturer's protocol. Stable cell lines expressing IQGAP1 or IQGAP1 siRNA and control cells were selected for 2 weeks with $0.4 \mathrm{mg} / \mathrm{ml} \mathrm{G} 418$ sulfate after transfection. The supernatant from cells of IQGAP1-overexpressing or knockdown and control was collected as conditioned medium for tube formation of HUVECs and chicken embryo chorioallantoic membrane (CAM) assays (described below). For AKT inhibitor (LY294002) or ERK inhibitor (PD98059) (Calbiochem, Merck Biosciences, Merck KGaA, Darmstadt, Germany) treatment, IQGAP1-overexpressing cells were seeded and after $24 \mathrm{~h}$, cells were incubated with $20 \mu \mathrm{M}$ LY294002 or PD98059 in the absence of serum for $48 \mathrm{~h}$. The supernatant was collected as conditioned medium for tube formation of HUVECs and chicken embryo CAM assays (described below).

Western blot analysis. The proteins in the cell lysates were quantified using the Bradford method. Proteins (70 $\mu \mathrm{g})$ were processed by electrophoretic separation on $10 \%$ SDS-PAGE and transferred to a nitrocellulose membrane, which was then blocked with PBS/Tween-20 containing 5\% non-fat milk. The membranes were incubated overnight at $4^{\circ} \mathrm{C}$ with corresponding primary antibodies. To normalize protein loading, mouse anti- $\beta$-actin antibody (1:5,000; cat. no. A5441; Sigma-Aldrich; Merck KGaA, Darmstadt, Germany) was used. After incubation with HRP-conjugated secondary antibody for $2 \mathrm{~h}$ at room temperature, target proteins on the membrane were visualized using an enhanced chemiluminescence (ECL) detection system (Beijing ComWin Biotech Co., Ltd, Beijing, China). The band intensity was analyzed using Bio-Rad's Image Lab software (Bio-Rad Laboratories, Inc., Hercules, CA, USA). Mouse anti-IQGAP1 antibody was purchased from BD Biosciences (1:5,000; cat. no. 610612; Franklin Lakes, NJ, USA). Rabbit anti-GFP (1:1,000; cat. no. D110008) and rabbit anti-VEGFR2 (1:500; cat. no. D151118) antibodies were purchased from Sangon Biotech Company (Shanghai, China). Rabbit anti-VEGF (1:500; cat. no. A12303) and rabbit anti-p-VEGFR2 (1:500; cat. no. AP0382) antibodies were purchased from ABclonal Biotechnology Co., Ltd. (Wuhan, China). Rabbit anti-p-AKT (1:1,000; cat. no. 4060), total rabbit anti-AKT (1:1,000; cat. no. 9272), rabbit anti-p-ERK1/2 (1:1,000; cat. no. 9101) and total rabbit anti-ERK1/2 (1:1,000; cat. no. 4695) were purchased from Cell Signaling Technology, Inc. (Danvers, MA, USA).

Tube formation assay. A total of $50 \mu \mathrm{l}$ of chilled Matrigel (BD Biosciences) was added to a 96-well plate and incubated at $37^{\circ} \mathrm{C}$ for $30 \mathrm{~min}$. HUVECs $\left(1 \times 10^{4}\right)$ in $100 \mu \mathrm{l}$ of conditioned medium were seeded onto each well and incubated at $37^{\circ} \mathrm{C}$ in $5 \% \mathrm{CO}_{2}$ for $6 \mathrm{~h}$. Images were captured by phase-contrast microscopy and the tubular structures were quantified by manual counting in three random fields per well to obtain the sum.

Chicken embryo CAM assay. Seven-day-old chicken embryos were windowed to expose the CAM and the conditioned medium was placed onto the CAM. The windows were sealed with cellophane tape and embryos were transferred back into the incubator. After 3 days, chicken embryos were fixed with stationary solution (methanol:acetone 1:1) for $15 \mathrm{~min}$, CAMs were cut and harvested, and then photographed. The ability of angiogenesis in chicken embryo CAM was quantitated by measuring the number of vessels.

Statistical analysis. Statistical analysis was conducted using the SPSS 17.0 software package (SPSS, Inc., Chicago, IL, USA). All experiments were performed in triplicate. Data are presented as the mean \pm SD. The differences between groups were assessed by one-way ANOVA and followed up using Dunnett's multiple comparison post hoc test. $\mathrm{P}<0.05$ was considered to indicate a statistically significant result.

\section{Results}

Generation of stable IQGAPI-overexpressing and silenced clones in the ESCC cell lines. In order to obtain insight into the effect of IQGAP1 on tumor angiogenesis, we overexpressed IQGAP1 in human ESCC EC9706 cells. Western blot analysis results showed that stable clones transfected with the expression vector carrying cDNA for human full-length IQGAP1 (named as IQ-1 and IQ-2) expressed fusion protein 
A
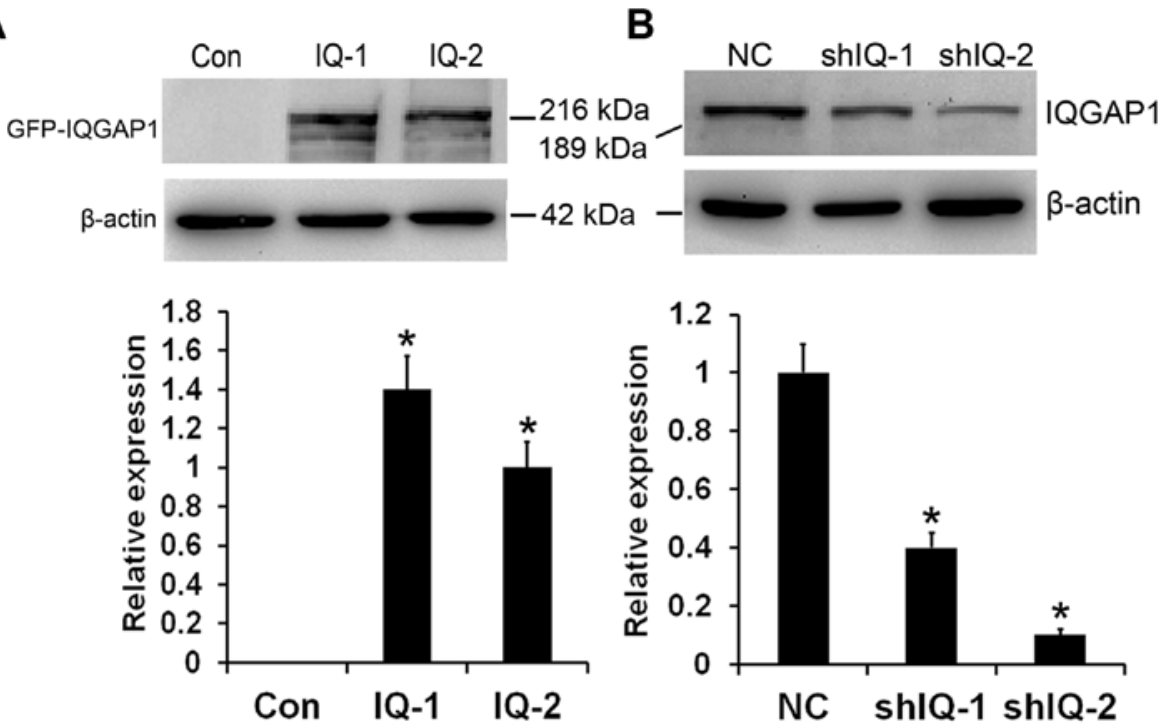

Figure 1. The establishment of stable IQGAP1-overexpressing and -knockdown ESCC cell lines. (A) EC9706 cells were stably transfected with the control (Con) or pEGFP-IQGAP1 expressing vectors (IQ-1 and IQ-2), and clones expressing a transfected GFP-IQGAP1 protein were analyzed by western blot analysis. The histogram represents quantitative densitometry of the proteins. Data are presented as mean $\pm \mathrm{SD}, \mathrm{n}=3,{ }^{*} \mathrm{P}<0.01$, compared with the control cells. (B) KYSE150 cells were stably transfected with the control (NC) or IQGAP1-shRNA vectors (shIQ-1 and shIQ-2), and IQGAP1 protein expression in the control and IQGAP1-knockdown stable cell lines was assessed by western blot analysis. The histogram represents quantitative densitometry of proteins. Data are presented as mean $\pm \mathrm{SD}, \mathrm{n}=3,{ }^{*} \mathrm{P}<0.01$, compared with the control cells. IQGAP1, IQ-domain GTPase activating protein 1.

A

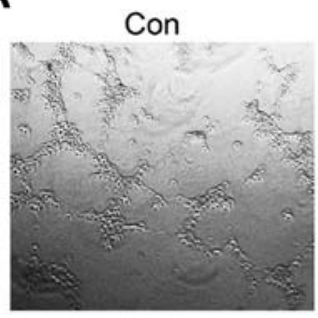

B

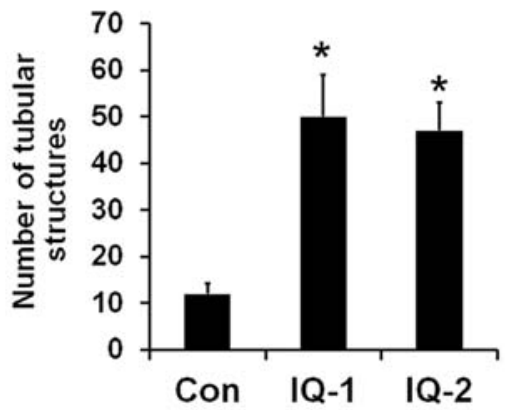

C

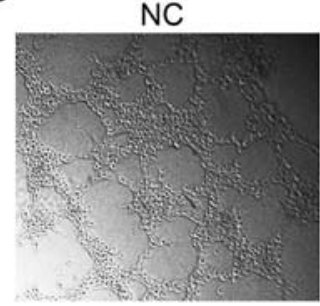

IQ-1

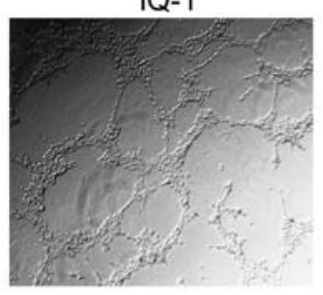

IQ-2

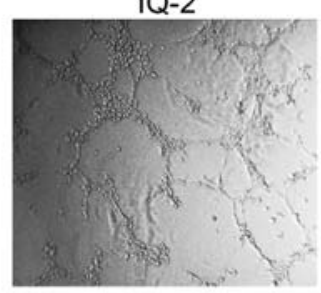

D

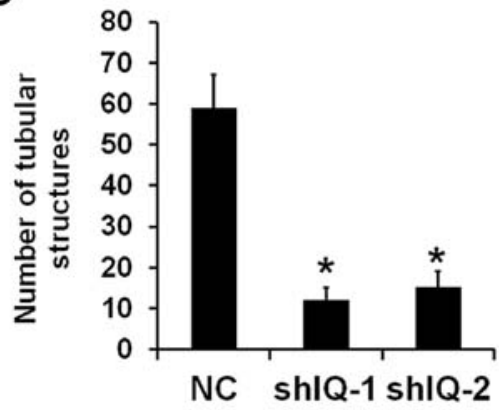

Figure 2. Effect of IQGAP1 on the tube formation of human umbilical vascular endothelial cells (HUVECs). (A) Representative results of HUVEC tube formation cultured on Matrigel-coated plates with conditioned media from the IQGAP1-overexpressing (IQ-1 and IQ-2) and control (Con) cells. (B) The number of tubular structures was counted in each group. Data are presented as mean $\pm \mathrm{SD}, \mathrm{n}=3,{ }^{*} \mathrm{P}<0.01$, compared with the control cells. (C) Representative results of HUVEC tube formation cultured on Matrigel-coated plates with conditioned media from the IQGAP1 knockdown (shIQ-1 and shIQ-2) and control (NC) cells. (D) The number of tubular structures was counted in each group. Data are presented as mean $\pm \mathrm{SD}, \mathrm{n}=3,{ }^{*} \mathrm{P}<0.01$, compared with the control cell. IQGAP1, IQ-domain GTPase activating protein 1. 
A

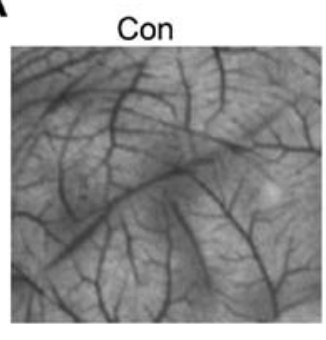

IQ-1

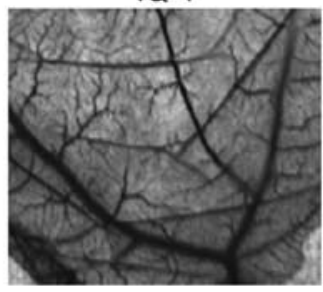

IQ-2

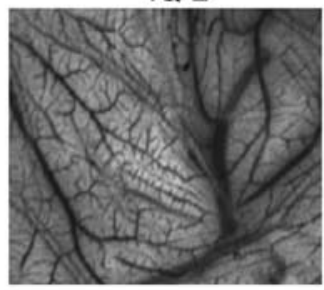

B

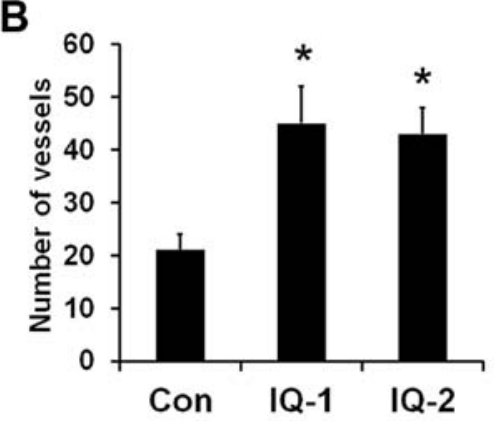

C

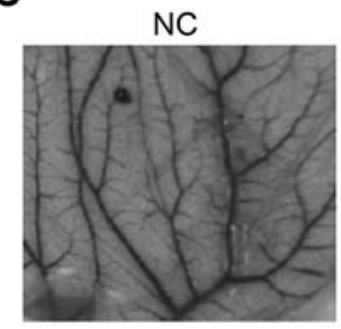

D

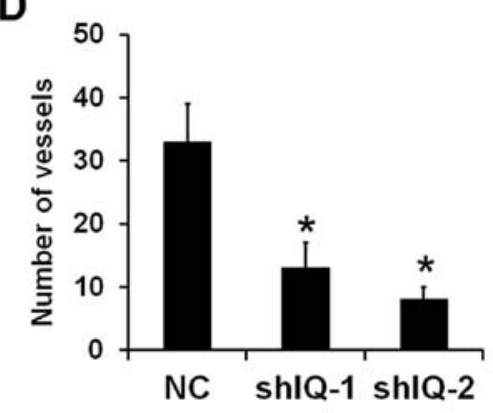

Figure 3. Effect of IQGAP1 on the angiogenesis in the chicken embryo CAM assay. (A) Representative images of the blood vessels formed in the CAM assay with conditioned media from the IQGAP1 overexpression (IQ-1 and IQ-2) and control (Con) cells. (B) The number of vessels in each group was counted. Data are presented as mean $\pm \mathrm{SD}, \mathrm{n}=3,{ }^{*} \mathrm{P}<0.01$, compared with the control cells. (C) Representative images of the blood vessels formed in the CAM assay with conditioned media from the IQGAP1 knockdown and (shIQ-1 and shIQ-2) and control (NC) cells. (D) The number of vessels in each group was counted. Data are presented as mean $\pm \mathrm{SD}, \mathrm{n}=3,{ }^{*} \mathrm{P}<0.01$, compared with the control cells. IQGAP1, IQ-domain GTPase activating protein 1 .

of GFP-IQGAP1, which was not found in the control vector (named as Con) (Fig. 1A). To further evaluate the roles of IQGAP1 in tumor angiogenesis and the potential of IQGAP1 downregulation for ESCC therapy, IQGAP1 stable knockdown was performed in the human ESCC KYSE150 cell line. As shown in Fig. 1B, two stable clones (named as shIQ-1 and shIQ-2) exhibited efficiently reduced expression levels of IQGAP1 protein compared with the control cells (named as NC).

IQGAPI overexpression promotes tube formation of HUVECs. The tube formation assay can mimic certain stages of angiogenesis, which is a well-established in vitro angiogenesis test $(15,16)$. To assess the functional role of IQGAP1 in angiogenesis in vitro, we investigated whether IQGAP1 is involved in capillary tube formation. As shown in Fig. 2A, the HUVECs spontaneously formed capillary-like tube structures after $6 \mathrm{~h}$ of incubation on Matrigel. The conditioned medium from IQGAP1 overexpressing cells increased the number of capillary-like structures. Quantification of the number of tubular structure showed that IQGAP1 overexpression resulted in a 5-to 6-fold increasing in tube formation by HUVECs (Fig. 2B). In contrast, IQGAP1 knockdown resulted in less elongated, broken and foreshortened tubes compared to the control shRNA-transfected cells (Fig. 2C). An approximate 6-to 7-fold decrease in tube formation was observed in the IQGAP1 shRNA-transfected cells (Fig. 2D). As a consequence, this finding confirmed that IQGAP1 functions as a promoter of tumor angiogenesis in vitro.

IQGAPI overexpression stimulates angiogenesis in the chicken embryo CAMassay. Chicken embryo CAM assay is a well-known model of angiogenesis that can be widely used to investigate new vessel formation and inhibition in vivo (17). To further evaluate the potential effect of IQGAP1 on angiogenesis, chicken embryo CAM assay was employed. The results showed that IQGAP1 overexpression induced a stronger proangiogenic response in a chicken embryo CAM assay than the control (Fig. 3A). The number of branches of microvessels in the conditioned medium from the IQGAP1-overexpressing cells increased to 2.5-to 3-fold of the control (Fig. 3B). Conversely, IQGAP1 knockdown inhibited angiogenesis (Fig. 3C). Quantitative analysis revealed that conditioned medium from the IQGAP1-knockdown cells caused a 3.5-to 4-fold reduction in the number of blood vessels (Fig. 3D). These results further confirm that IQGAP1 overexpression induces tumor angiogenesis in vivo. 
A
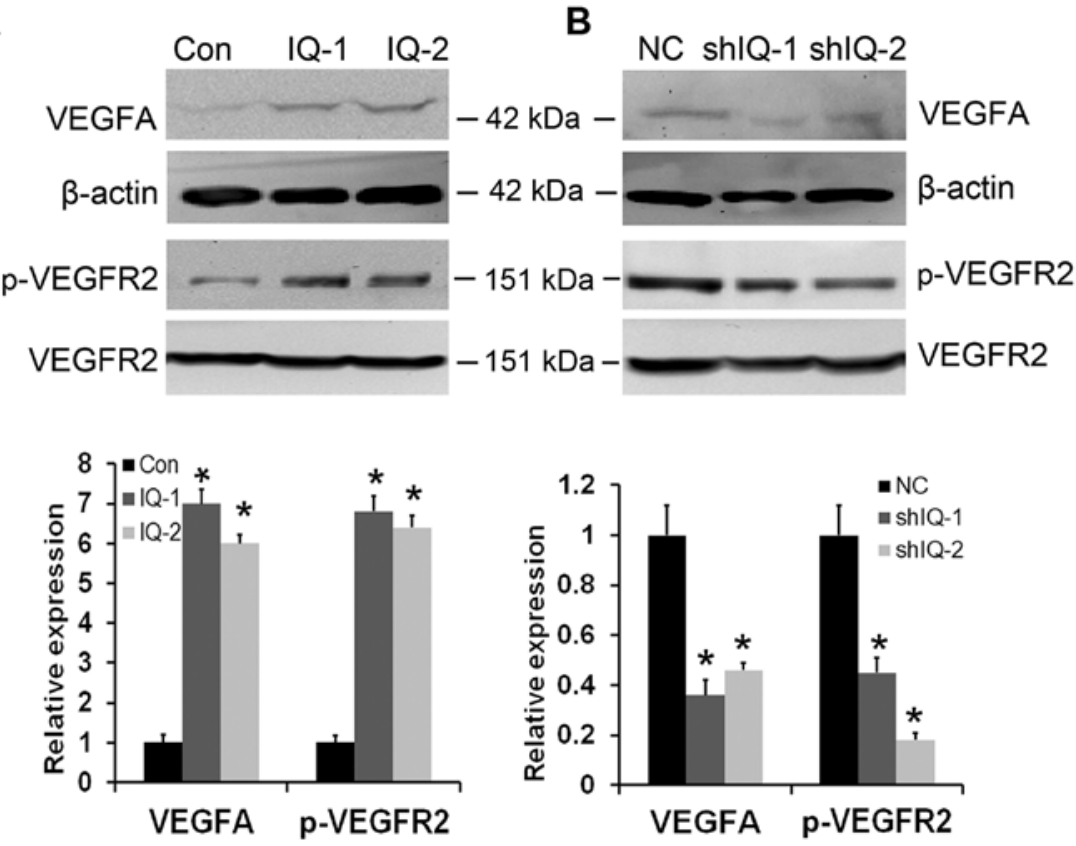

Figure 4. IQGAP1 regulates the VEGF-VEGFR2 signaling pathway. (A) IQGAP1 overexpression (IQ-1 and IQ-2) resulted in increased the protein levels of VEGF and phosphorylated (p)-VEGFR2. The histogram represents quantitative densitometry of proteins. Data are presented as mean $\pm \mathrm{SD}, \mathrm{n}=3$, ${ }^{*} \mathrm{P}<0.01$, compared with the control cells. (B) IQGAP1 knockdown (shIQ-1 and shIQ-2) downregulated the protein levels of VEGF and p-VEGFR2. The histogram represents quantitative densitometry of proteins. Data are presented as mean $\pm \mathrm{SD}, \mathrm{n}=3,{ }^{*} \mathrm{P}<0.01$, compared with the control cells. IQGAP1, IQ-domain GTPase activating protein 1; VEGF, vascular endothelial growth factor; VEGFR2, vascular endothelial growth factor receptor 2.

A

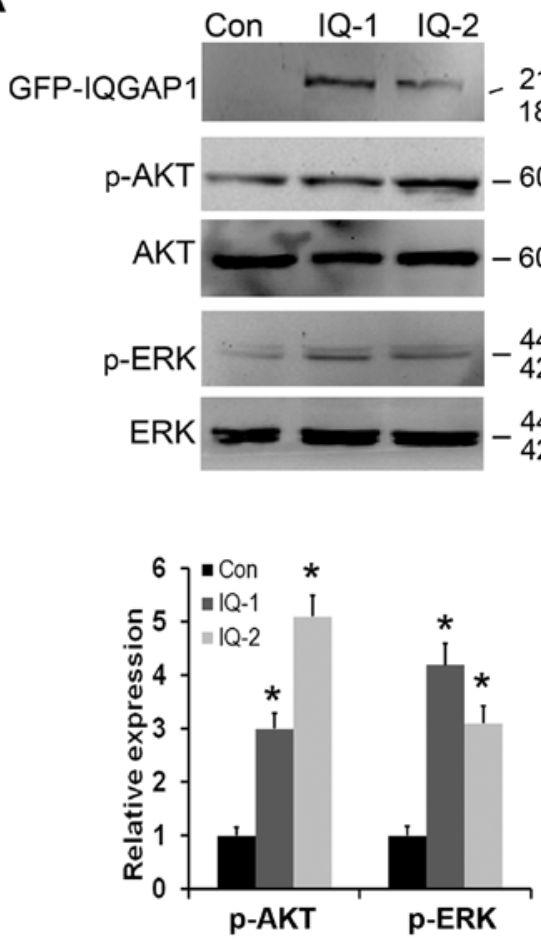

B

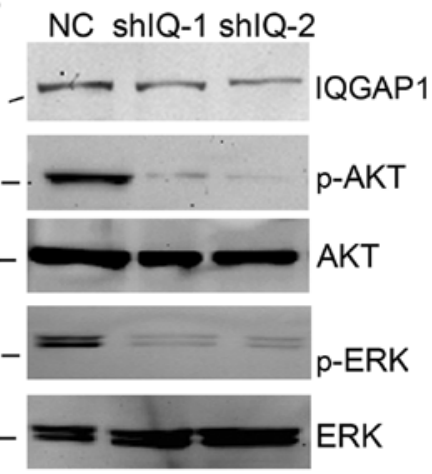

Figure 5. IQGAP1 regulates AKT and ERK activity. (A) IQGAP1 overexpression (IQ-1 and IQ-2) obviously increased the protein levels of phosphorylated (p)-AKT and p-ERK, whereas the levels of total AKT and ERK protein did not change. The histogram represents quantitative densitometry of proteins. Data are presented as mean $\pm \mathrm{SD}, \mathrm{n}=3,{ }^{*} \mathrm{P}<0.01$, compared with the control cells. (B) IQGAP1 knockdown (shIQ-1 and shIQ-2) obviously resulted in decreased p-AKT and p-ERK, whereas the levels of total ERK and AKT were not altered. The histogram represents quantitative densitometry of proteins. Data are presented as mean $\pm \mathrm{SD}, \mathrm{n}=3,{ }^{*} \mathrm{P}<0.01$, compared with the control cells. IQGAP1, IQ-domain GTPase activating protein 1 .

IQGAPI overexpression enhances expression of VEGF and activation of VEGFR2. Considering that angiogenic factor VEGF is a prime regulator of angiogenesis (18), we first examined the expression of VEGF in IQGAP1-overexpressing and -silenced ESCC cells. As shown in Fig. 4A and B, IQGAP1 overexpression upregulated the expression levels of 
A

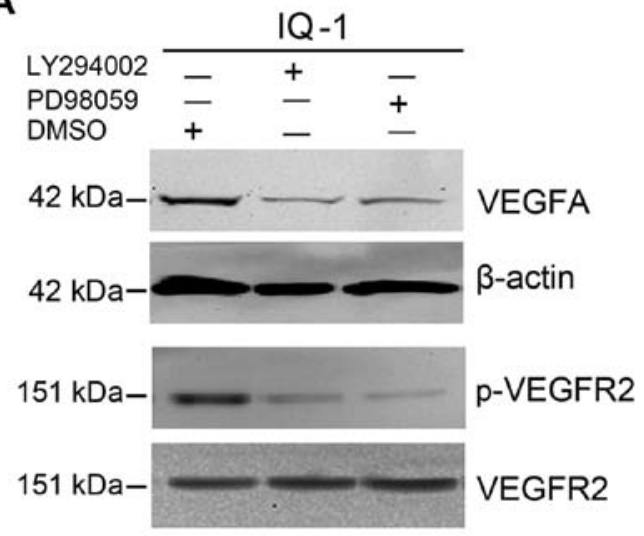

B

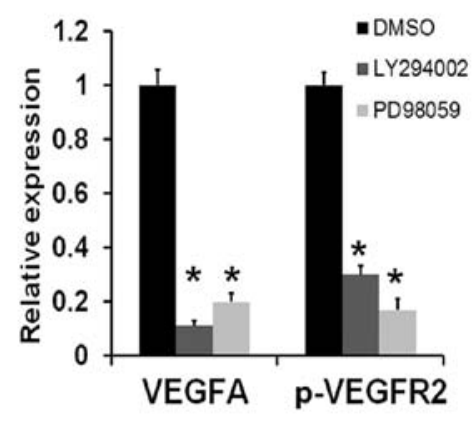

C

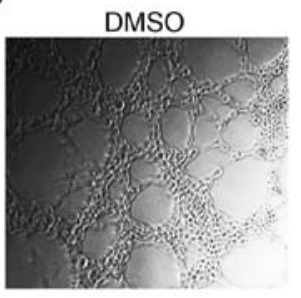

LY294002

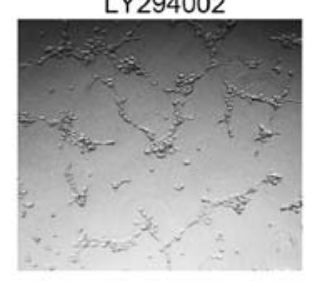

PD98059

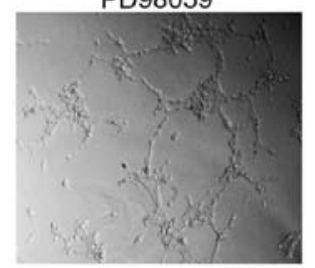

D

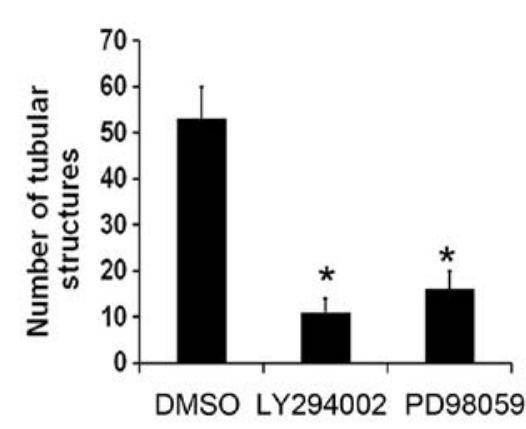

$\mathbf{F}$

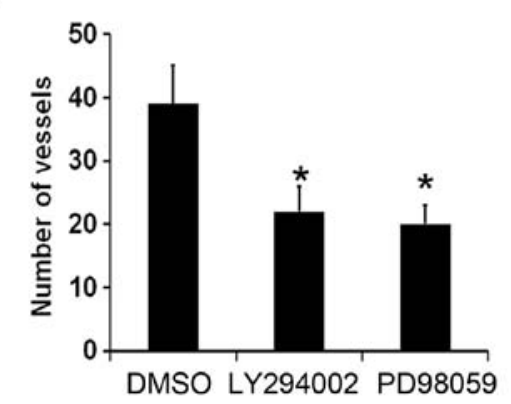

E

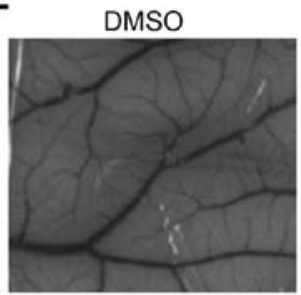

LY294002

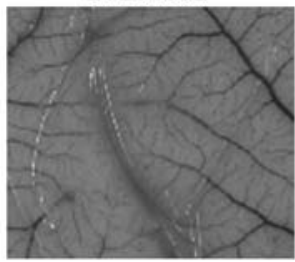

PD98059

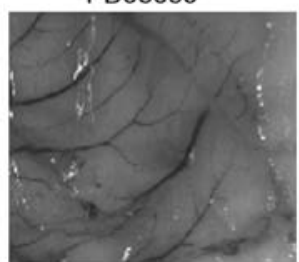

Figure 6. IQGAP1 regulates expression of VEGF and phosphorylated (p)-VEGFR2 as well as angiogenesis through AKT and ERK signaling. (A) AKT inhibitor (LY294002) or ERK inhibitor (PD98059) $(20 \mu \mathrm{M})$ was used to treat IQGAP1-overexpressing cells (IQ-1) for $48 \mathrm{~h}$, and abrogated the effects of IQGAP1 overexpression-mediated upregulation of VEGFA and p-VEGFR2. (B) The histogram represents quantitative densitometry of proteins. Data are presented as mean $\pm \mathrm{SD}, \mathrm{n}=3,{ }^{*} \mathrm{P}<0.01$, compared with the control cells (DMSO). (C) The promoting effect of IQGAP1 overexpression on HUVEC tube formation was attenuated by the AKT or ERK inhibitor. (D) The number of tubular structures was counted in each group. Data are presented as mean $\pm \mathrm{SD}, \mathrm{n}=3$, ${ }^{*} \mathrm{P}<0.01$, compared with the control cells (DMSO). (E) The pro-angiogenic function of IQGAP1 overexpression in chicken embryo CAM was abrogated when IQGAP1 overexpression cells were treated with AKT or ERK inhibitor. (F) The number of vessels in each group was counted. Data are presented as mean \pm SD, $n=3$, "P<0.01, compared with the control cells (DMSO). IQGAP1, IQ-domain GTPase activating protein 1; VEGF, vascular endothelial growth factor; VEGFR2, vascular endothelial growth factor receptor 2 .

VEGF, whereas VEGF expression was obviously decreased in the IQGAP1-silenced. VEGFR2 is the most biologically important receptor for VEGF (19). Thus, we subsequently examined VEGFR2 expression and activation in the IQGAP1-overexpressing and -silenced ESCC cells. IQGAP1 overexpression significantly enhanced phosphorylation of VEGFR2, without obviously affecting overall VEGFR2 expression levels. Conversely, IQGAP1 knockdown inhibited the phosphorylation of VEGFR2, while the total levels of VEGFR2 had little change (Fig. 4A and B). These results clearly demonstrate that IQGAP1 overexpression can promote tumor angiogenesis by upregulating VEGF-VEGFR2 signaling.
IQGAPI overexpression promotes tumor angiogenesis through AKT and ERK activation. To identify the potential molecular mechanisms of IQGAP1 in tumor angiogenesis, we analyzed the expression levels of various signaling proteins by western blot assay. The results showed that IQGAP1 overexpression markedly increased the levels of p-AKT and p-ERK, whereas the levels of total AKT and ERK were not altered (Fig. 5A). Silencing of IQGAP1 expression led to a significant decrease in the expression of p-AKT and p-ERK proteins, and had no effect on total AKT and ERK protein expression (Fig. 5B). To determine whether the IQGAP1-mediated increase in VEGF and p-VEGFR2 expression as well as tumor angiogenesis is mediated by regulating AKT or ERK signaling, we analyzed 
the effect of specific AKT and ERK inhibitors (LY294002 or PD98059) on IQGAP1-overexpressing cells. As shown in Fig. 6A and B, the AKT and ERK inhibitor abolished the role of IQGAP1 overexpression on VEGF and p-VEGFR2 upregulation. Furthermore, we found that LY294002 or PD98059 could abrogate the effects of IQGAP1-mediated tumor angiogenesis by in vitro tube formation of HUVECs (Fig. 6C and D) and in vivo chicken embryo CAM assay (Fig. $6 \mathrm{E}$ and F). Taken together, these observations demonstrate that IQGAP1 overexpression promotes tumor angiogenesis by targeting the AKT and ERK-mediated VEGF-VEGFR2 signaling pathway.

\section{Discussion}

Esophageal squamous cell carcinoma (ESCC) is one of the leading causes of cancer-related death due to the high incidence of advanced disease, metastasis, and resistance to radiotherapy and chemotherapy (1). Thus, it is urgent to identify novel targets and new strategies to treat this disease. Angiogenesis plays a significant role in the continuous growth of tumors, invasion and metastasis as capillary formation in tumors can provide nutrients and oxygen to supply the growing tumor and also act as conduits for the metastasis of tumors $(2,3,19)$. Consequently, more and more attention has been focused on tumor angiogenesis; and thus, anti-angiogenic therapy has become one of the most promising and efficient strategy for inhibiting tumor growth and progression.

The development of ESCC involves the accumulation of the abnormal expression of oncogenes involved in the initiation and progression of ESCC. IQGAP1 is a member of the IQGAP family of multidomain proteins $(6,20)$. Cumulative evidence suggests that IQGAP1 is an oncogene and is overexpressed in several types of human cancers $(4,21)$. Consistent with these findings, we reported that IQGAP1 is upregulated in ESCC tissues and is correlated with the invasive depth of ESCC tumors (14). However, it has not yet been elucidated whether IQGAP1 is involved in tumor angiogenesis in ESCC during which IQGAP1 is upregulated. In the present study, we found that IQGAP1 overexpression significantly increased the angiogenesis confirmed by HUVEC tube formation assay in vitro and chicken embryo CAM assay in vivo, whereas the angiogenesis ability was markedly suppressed when IQGAP1 expression was silenced. These results indicate that IQGAP1 is an attractive molecule for targeting tumor angiogenesis against cancer progression.

Angiogenesis is a complex multistep process which is regulated by several endogenous angiogenic activators and inhibitors $(2,3,22)$. Of the numerous endogenous proangiogenic factors, VEGF is well known as a key regulator of the process of tumor angiogenesis by stimulating endothelial cell proliferation, migration and invasion. VEGF exerts its biological effects by binding to specific tyrosine kinase receptors on the cell surface, called VEGF receptors (VEGFRs), and VEGFR2 is the major mediator of VEGF-induced angiogenesis. The binding of VEGF to VEGFR2 leads to the intrinsic tyrosine kinase activation of the receptors followed by dimerization and autophosphorylation of VEGFR2, and then triggers a downstream signaling cascade $(19,23,24)$. Therefore, we hypothesized that IQGAP1 regulates ESCC angiogenesis by regulating the VEGF-VEGFR2 pathway. In the present study, we observed that overexpression of IQGAP1 strongly increased VEGF expression and phosphorylation of VEGFR2, while knockdown of IQGAP1 obviously decreased VEGF and p-VEGFR2 expression. These findings showed that IQGAP1 could regulate tumor angiogenesis by controlling the activation of VEGF-VEGFR2 signaling. It has been reported that IQGAP1 can directly bind to VEGFR2 and also is necessary for VEGF to stimulate angiogenesis in MCF-7 and HUVECs (9,25-27), which is consistent with our findings. Considering that the VEGF-VEGFR signaling pathway is a significant factor underlying angiogenesis, numerous therapeutic strategies have been developed to target angiogenesis by blocking this pathway. Accordingly, this study further indicates that targeting IQGAP1 represents a promising therapeutic strategy for tumor angiogenesis.

AKT and ERK are serine/threonine kinases that are critical for many diverse processes, including cell proliferation, apoptosis, migration, angiogenesis and metastasis (28-31). IQGAP1, as a scaffold protein, contains multiple domains which mediate binding to a number of proteins. It has been reported that IQGAP1 can combine with AKT and ERK and regulate their activity $(6,21,32-35)$. To explore the potential pro-angiogenic mechanisms of IQGAP1 in ESCC, we detected the expression of the AKT and ERK signaling pathway. The data showed that IQGAP1 overexpression could increase phosphorylation of AKT and ERK. Moreover, IQGAP1 knockdown could inhibit AKT and ERK activation. Furthermore, we observed that AKT and ERK inhibitors significantly decreased VEGF expression and VEGFR2 phosphorylation in IQGAP1-overexpressing cells. Moreover, the pro-angiogenic effect of IQGAP1 overexpression on angiogenesis in tube formation of HUVECs and a chick embryo CAM angiogenesis model was abrogated when IQGAP1-overexpressing cells were treated with the AKT and ERK inhibitor. These findings suggest that IQGAP1 promotes tumor angiogenesis mainly via AKT or ERK/VEGF-VEGFR2 signaling pathway.

In summary, we demonstrated for the first time that IQGAP1 overexpression could promote angiogenesis in ESCC by targeting the AKT or ERK/VEGF-VEGFR2 signaling pathway. Moreover, silencing of the expression of IQGAP1 inhibited tumor angiogenesis. Our studies not only demonstrated that IQGAP1 regulated the tumor angiogenesis of ESCC, but also revealed a therapeutic opportunity in targeting IQGAP1 for cancer treatment.

\section{Acknowledgements}

Not applicable.

\section{Funding}

The present study was supported by the National Natural Science Foundation of China (no. 81372676) and the Natural Science Foundation of Shanxi Province (no. 201601D011130).

\section{Availability of data and material}

All data generated or analyzed during this study are included in this published article. 


\section{Authors' contributions}

CHL and XJS carried out the experiments and interpreted the data. SSN, CYY, YPH and JTK participated in the collection of the data. XXW and XZL designed the research, supervised the study, interpreted data and wrote the manuscript. All authors read and approved the manuscript and agree to be accountable for all aspects of the research in ensuring that the accuracy or integrity of any part of the work are appropriately investigated and resolved.

\section{Ethics approval and consent to participate}

Not applicable.

\section{Patient consent for publication}

Not applicable.

\section{Competing interests}

The authors declare that they have no competing interests.

\section{References}

1. Chen W, Zheng R, Baade PD, Zhang S, Zeng H, Bray F, Jemal A, $\mathrm{Yu}$ XQ and He J: Cancer statistics in China, 2015. CA Cancer J Clin 66: 115-132, 2016

2. Carmeliet P: Angiogenesis in life, disease and medicine. Nature 438: 932-936, 2005.

3. Folkman J: Angiogenesis. Annu Rev Med 57: 1-18, 2006.

4. Johnson M, Sharma M and Henderson BR: IQGAP1 regulation and roles in cancer. Cell Signal 21: 1471-1478, 2009.

5. Noritake J, Watanabe T, Sato K, Wang S and Kaibuchi K IQGAP1: A key regulator of adhesion and migration. J Cell Sci 118: 2085-2092, 2005.

6. White CD, Erdemir HH and Sacks DB: IQGAP1 and its binding proteins control diverse biological functions. Cell Signal 24: 826-834, 2012.

7. Nabeshima K, Shimao $Y$, Inoue $T$ and Koono $M$ Immunohistochemical analysis of IQGAP1 expression in human colorectal carcinomas: Its overexpression in carcinomas and association with invasion fronts. Cancer Lett 176: 101-109, 2002.

8. Hayashi H, Nabeshima K, Aoki M, Hamasaki M, Enatsu S, Yamauchi Y, Yamashita Y and Iwasaki H: Overexpression of IQGAP1 in advanced colorectal cancer correlates with poor prognosis-critical role in tumor invasion. Int J Cancer 126 2563-2574, 2010

9. Jadeski L, Mataraza JM, Jeong HW, Li Z and Sacks DB: IQGAP1 stimulates proliferation and enhances tumorigenesis of human breast epithelial cells. J Biol Chem 283: 1008-1017, 2008.

10. Dong P, Nabeshima K, Nishimura N, Kawakami T, Hachisuga T, Kawarabayashi $\mathrm{T}$ and Iwasaki H: Overexpression and diffuse expression pattern of IQGAP1 at invasion fronts are independent prognostic parameters in ovarian carcinomas. Cancer Lett 243: 120-127, 2006.

11. Zhao H, Xie C, Lin X, Zhao Y, Han Y, Fan C, Zhang X, Du J, Han Y, Han Q, et al: Coexpression of IQ-domain GTPase-activating protein 1 (IQGAP1) and Dishevelled (Dvl) is correlated with poor prognosis in non-small cell lung cancer PLoS One 9: e113713, 2014.

12. Wang XX, Li XZ, Zhai LQ, Liu ZR, Chen XJ and Pei Y: Overexpression of IQGAP1 in human pancreatic cancer. Hepatobiliary Pancreat Dis Int 12: 540-545, 2013.

13. Liu Z, Liu D, Bojdani E, El-Naggar AK, Vasko V and Xing M: IQGAP1 plays an important role in the invasiveness of thyroid cancer. Clin Cancer Res 16: 6009-6018, 2010.
14. Wang XX, Wang K, Li XZ, Zhai LQ, Qu CX, Zhao Y, Liu ZR, Wang HZ, An QJ, Jing LW, et al: Targeted knockdown of IQGAP1 inhibits the progression of esophageal squamous cell carcinoma in vitro and in vivo. PLoS One 9: e96501, 2014.

15. Arnaoutova I, George J, Kleinman HK and Benton G: The endothelial cell tube formation assay on basement membrane turns 20: State of the science and the art. Angiogenesis 12: 267-274, 2009.

16. Ponce ML: Tube formation: An in vitro matrigel angiogenesis assay. Methods Mol Biol 467: 183-188, 2009.

17. Ribatti D, Vacca A, Roncali L and Dammacco F: The chick embryo chorioallantoic membrane as a model for in vivo research on angiogenesis. Int J Dev Biol 40: 1189-1197, 1996.

18. Verheul HM and Pinedo HM: The role of vascular endothelial growth factor (VEGF) in tumor angiogenesis and early clinical development of VEGF-receptor kinase inhibitors. Clin Breast Cancer 1 (Suppl 1): S80-S84, 2000.

19. Zetter BR: Angiogenesis and tumor metastasis. Annu Rev Med 49: 407-424, 1998.

20. Osman M: An emerging role for IQGAP1 in regulating protein traffic. ScientificWorldJournal 10: 944-953, 2010.

21. White CD, Brown MD and Sacks DB: IQGAPs in cancer: A family of scaffold proteins underlying tumorigenesis. FEBS Lett 583: 1817-1824, 2009.

22. Detmar M: Tumor angiogenesis. J Investig Dermatol Symp Proc 5: 20-23, 2000

23. Shibuya M: Vascular endothelial growth factor (VEGF) and its receptor (VEGFR) signaling in angiogenesis: A crucial target for anti- and pro-angiogenic therapies. Genes Cancer 2: 1097-1105, 2011.

24. McMahon G: VEGF receptor signaling in tumor angiogenesis. Oncologist 5 (Suppl 1): S3-S10, 2000.

25. Yamaoka-Tojo M, Tojo T, Kim HW, Hilenski L, Patrushev NA, Zhang L, Fukai T and Ushio-Fukai M: IQGAP1 mediates VE-cadherin-based cell-cell contacts and VEGF signaling at adherence junctions linked to angiogenesis. Arterioscler Thromb Vasc Biol 26: 1991-1997, 2006

26. Yamaoka-Tojo M, Ushio-Fukai M, Hilenski L, Dikalov SI, Chen YE, Tojo T, Fukai T, Fujimoto M, Patrushev NA, Wang $\mathrm{N}$, et al: IQGAP1, a novel vascular endothelial growth factor receptor binding protein, is involved in reactive oxygen species-dependent endothelial migration and proliferation. Circ Res 95: 276-283, 2004.

27. Meyer RD, Sacks DB and Rahimi N: IQGAP1-dependent signaling pathway regulates endothelial cell proliferation and angiogenesis. PLoS One 3: e3848, 2008.

28. Fresno Vara JA, Casado E, de Castro J, Cejas P, Belda-Iniesta C and González-Barón M: PI3K/Akt signalling pathway and cancer. Cancer Treat Rev 30: 193-204, 2004.

29. Jiang BH and Liu LZ: AKT signaling in regulating angiogenesis. Curr Cancer Drug Targets 8: 19-26, 2008.

30. Dhillon AS, Hagan S, Rath O and Kolch W: MAP kinase signalling pathways in cancer. Oncogene 26: 3279-3290, 2007.

31. Ding C, Li L, Yang T, Fan X and Wu G: Combined application of anti-VEGF and anti-EGFR attenuates the growth and angiogenesis of colorectal cancer mainly through suppressing AKT and ERK signaling in mice model. BMC Cancer 16: 791, 2016.

32. Sbroggiò M, Carnevale D, Bertero A, Cifelli G, De Blasio E, Mascio G, Hirsch E, Bahou WF, Turco E, Silengo L, et al: IQGAP1 regulates ERK1/2 and AKT signalling in the heart and sustains functional remodelling upon pressure overload. Cardiovasc Res 91: 456-464, 2011.

33. Chen F, Zhu HH, Zhou LF, Wu SS, Wang J and Chen Z: IQGAP1 is overexpressed in hepatocellular carcinoma and promotes cell proliferation by Akt activation. Exp Mol Med 42: 477-483, 2010.

34. Ma Y, Jin Z, Huang J, Zhou S, Ye H, Jiang S and Yu K: IQGAP1 plays an important role in the cell proliferation of multiple myeloma via the MAP kinase (ERK) pathway. Oncol Rep 30: 3032-3038, 2013.

35. Cheung KL, Lee JH, Shu L, Kim JH, Sacks DB and Kong AN: The Ras GTPase-activating-like protein IQGAP1 mediates Nrf2 protein activation via the mitogen-activated protein kinase/extracellular signal-regulated kinase (ERK) kinase (MEK)-ERK pathway. J Biol Chem 288: 22378-22386, 2013. 